\title{
Anomie Warum sehen wir nicht die Ordnung in der Unordnung?
}

Syrien, Jemen, Afghanistan, Irak, Somalia - unser Blick auf Krisenräume richtet sich fast ausschließlich auf deren Anomie: den Zusammenbruch sozialer Normen, Regeln und Ordnungsstrukturen, die endemische Gewalt, die (scheinbare) Anarchie. Bekanntlich hat Thomas Hobbes einst die Metapher des Naturzustandes entwickelt, um ein Bild der Herrschaftslosigkeit zu zeichnen, in der es notwendigerweise zum Krieg aller gegen alle komme, weil eine zentrale Instanz zur Konfliktregelung fehle. Mir scheint, Hobbes' Geist trübt bis heute unseren Blick, indem er in diesen Krisenräume die Logik des Naturzustands am Werk sieht. Was aber ist mit den vielfältigen Aktivitäten, die zur Aufrechterhaltung einer»Ordnung in der Unordnung « unternommen werden, die oft rudimentäre öffentliche Dienstleistungen und damit das Überleben vieler Menschen ermöglichen - inmitten scheinbarer Herrschaftslosigkeit und über Kampflinien hinweg. Wir wissen noch wenig, wann, warum und wie solche Ordnungsversuche gelingen, wann sie scheitern und wie sie in die Konfiguration von Gewalt und Krieg eingebunden sind und diese möglicherweise reproduzieren. Wann fangen wir an, uns für die oft verdeckten, dabei nicht weniger spektakulären und zugleich ambivalenten Taten der»Helden ohne Waffen«zu interessieren? 